\title{
Civilisations
}

Revue internationale d'anthropologie et de sciences

humaines

58-2 | 2009

Intimités et inimitiés du religieux et du politique en

Afrique

\section{Le kimbanguisme et le pouvoir en RDC}

entre apolitisme et conception théologico-politique

\section{Anne Mélice}

\section{(2) OpenEdition}

\section{Journals}

Édition électronique

URL : http://journals.openedition.org/civilisations/2035

DOI : $10.4000 /$ civilisations. 2035

ISSN : 2032-0442

Éditeur

Institut de sociologie de l'Université Libre de Bruxelles

Édition imprimée

Date de publication : 30 décembre 2009

Pagination : $59-80$

ISBN : 2-87263-027-9

ISSN : 0009-8140

Référence électronique

Anne Mélice, «Le kimbanguisme et le pouvoir en RDC », Civilisations [En ligne], 58-2 | 2009, mis en

ligne le 30 décembre 2012, consulté le 02 mai 2019. URL : http://journals.openedition.org/

civilisations/2035; DOI : 10.4000/civilisations.2035

(C) Tous droits réservés 


\title{
Le kimbanguisme et le pouvoir en RDC entre apolitisme et conception théologico-politique
}

\author{
Anne MÉLICE
}

Résumé : . Cette étude a été suscitée par le constat d'une apparente inconséquence. De nombreux Kimbanguistes ont pris une part active, comme candidats de différents partis ou comme militants, aux élections présidentielles de 2006 en RDC. Or, le discours doctrinal de l'Église semble incompatible avec ces engagements dans la politique effective. D'abord, parce que l'Église affirme très tôt un apolitisme de principe. Ensuite, plus fondamentalement, parce que son discours doctrinal s'inscrit dans un horizon théologico-politique indifférent, voire hostile à la démocratie. C'est cette incompatibilité entre doctrine et pratique effective que nous cernerons ici. Nous verrons que l'écart entre le projet théologico-politique kimbanguiste, d'une part, la réalité politique actuelle, d'autre part, reste provisoire, et que c'est à l'histoire, conçue sur un mode eschatologique, qu'il appartiendrait de le résorber. En fait, aujourd'hui, le millénarisme qui supporte la doctrine kimbanguiste renvoie téléologiquement à plus tard la réalisation du projet théologico-politique kimbanguiste.

Mots-clés : kimbanguisme, EJCSK, Congo-Kinshasa, démocratie, apolitisme, conceptions théologico-politiques.

Abstract: The research reported in this paper was inspired by the observation of an apparent inconsistency. Many Kimbanguists took an active part in the presidential elections of 2006 in $D R C$, either as candidates of different parties or as militants, whereas Church doctrine seems to be incompatible with such a commitment in real politics. First, because the Kimbanguist Church affirmed from its beginnings an apolitical attitude. Second, and more fundamentally, because Kimbanguist theological-political doctrine is indifferent, if not hostile to democracy. It is this incompatibility between doctrine and real practice that I outline here. The article shows that the gap between the Kimbanguist theological-political project, on the one hand, and the current political reality, on the other hand, is temporary, and that it is up to history, conceived of in an eschatological perspective, to reduce it. In fact, the millenarianism that underlies the Kimbanguist doctrine postpones in a teleologic manner the realisation of the theological-political project.

Keywords: Kimbanguism, EJCSK, Congo-Kinshasa, democracy, apolitical attitude, theologicalpolitical conceptions. 


\title{
Introduction
}

\author{
A
}

vec l'institutionnalisation du kimbanguisme en Église en 1957-1958, la dimension politico-religieuse contestatrice de l'ordre établi du prophétisme initial disparaît dans une large mesure. L'apolitisme traverse désormais les déclarations de principe kimbanguistes. Toutefois, cet apolitisme s'accommode d'une certaine reconnaissance des pouvoirs politiques du Zaïre puis du Congo. Dans ce texte, nous montrerons que derrière l'apparente soumission à l'État et à ses dirigeants, d'autres positions, plus ambivalentes, relevant de l'imaginaire kimbanguiste ${ }^{1}$, sont apparues, dévoilant la complexité des rapports de l'EJCSK à la sphère politique.

Pour cerner cette complexité, la vision théologico-politique qui accompagne l'apolitisme kimbanguiste, ainsi que sa portée seront analysées. Cette perspective subordonne le pouvoir politique au pouvoir théologique et s'appuie ultimement sur la loi divine (Tosel 1995 : 180). Le contenu doctrinal du kimbanguisme que révèle la théologie kimbanguiste actuelle, présente des traits qui se resserrent autour du millénarisme et s'inscrit dans un horizon théologico-politique. Ainsi, nous montrerons qu'une tension habite le kimbanguisme. Si les Kimbanguistes prennent activement part à la vie politique, s'ils se présentent aux élections et le font sur les listes de partis différents, il demeure en effet que leur doctrine reste dominée par la perspective persistante d'un ordre théologico-politique sans mesure avec la réalité politique congolaise. Dans un ouvrage récent, Maurice Godelier a souligné « l'union, voire la fusion» (Godelier 2007 : 206) $\mathrm{du}$ politique et du religieux qu'on pouvait observer dans certains contextes. Le caractère fondamental des religions consiste, selon lui, à offrir « un fondement cosmique à un ordre social » (Godelier 2007 : 207). Les croyances, les rites, les statuts sociaux conférés à certains individus ou à certains groupes par la religion font jouer à celle-ci un rôle social qui trouve son expression culminante lorsque le politique et le religieux fusionnent en une seule personne, considérée comme « un dieu vivant parmi les hommes » (Godelier 2007 : 207). Or, chez les Kimbanguistes aujourd'hui, il est abondamment question d'un « Gouvernement de Dieu » à venir, dans lequel Kimbangu, divinisé, jouerait un rôle central.

D'un point de vue méthodologique, l'examen des rapports subtils de l'Église avec le pouvoir politique ne peut se limiter aux discours officiels des responsables de l'Église. Il importe en effet de faire aussi droit aux « relations complexes entre les représentations culturelles et les pratiques politiques, les modes populaires d'action politique, l'imaginaire politique » (Bayart 1996 : 9), en somme à ce que Jean-François Bayart a désigné comme « le politique par le bas ». L’Église kimbanguiste constitue l'un de ces « intermédiaires » permettant de mettre en lumière « les rapports de pouvoir « par le bas» plutôt que « par le haut»» (Bayart et al. 2008 : 10) et de dégager de la sorte « la face cachée des situations politiques » (Bayart et al. 2008 : 19). Dans ce texte, nous nous appuierons sur nos enquêtes

1. L'approche du politique par le bas fait droit au « rapport de la gouvernementalité à l'imaginaire, à la croyance [...] » (Bayart et al. $2008: 11-12$ ), à ce que Mbembe appelle « l'imaginaire du pouvoir, de l'autorité » (Mbembe $1988: 27)$. 
parmi les Kimbanguistes depuis $1995^{2}$, ainsi que sur une documentation spécifiquement kimbanguiste. Nous avons tenu à utiliser des " sources d'un autre type » que celles de la « science politique établie » (Bayart et al. 2008 : 11), particulièrement la prophétie, la révélation ou encore la parabole.

On trouvera en note quelques données démographiques ${ }^{3}$, géographiques ${ }^{4}$, financières et matérielles ${ }^{5}$ qui attestent le poids effectif de l'EJCSK.

Nous tirerons un fil historique. Mais il ne peut s'agir ici de développer la problématique complexe du caractère politique du mouvement kimbanguiste à l'époque coloniale (cf. Balandier 1982 ; Banda-Mwaka 1971 ; Mac Gaffey 1992 ; Young 1965). Sur le fond intangible d'un apolitisme de principe (1), on verra l'Église adopter successivement vis-à-vis des pouvoirs politiques des rapports divers : de contiguïté au pouvoir mobutiste (2.1), de défiance à l'égard du processus de démocratisation (2.2), de respect à l'égard des Kabila (3). Enfin, à l'égard du processus électoral, l'Église témoigne une attitude ambiguë (3). Notre intention est de porter à la réflexion l'incompatibilité apparente entre les positions doctrinales et les pratiques politiques effectives des membres de l'Église. Nous organiserons donc notre analyse autour de deux axes : le contenu doctrinal du kimbanguisme d'un côté, la pratique effective des Kimbanguistes de l'autre. Il s'agira, d'une part, de cerner le contenu résolument théologico-politique de la doctrine. Nous puiserons à cet effet aux discours de dignitaires kimbanguistes, mais aussi de fidèles. Nous ne nous en tiendrons pas à ces expressions de croyances partagées. On ne peut ignorer que celles-ci trouvent à se concrétiser symboliquement. La réalisation de la cité sainte de Nkamba constitue une expression sensible du projet théologico-politique. D'autre part, à côté de ces dimensions d'ordre imaginaire et symbolique, au sens de Godelier (Mélice 2007), nous interrogerons l'inscription du kimbanguisme dans le réel politique. Notre sentiment d'une incompatibilité entre les positions doctrinales et cette implication dans la réalité politique actuelle, n'est pas éprouvé par nos interlocuteurs comme une contradiction implacable. C'est, nous l'avancerons pour conclure, que cette apparente contradiction ressort d'une sorte de morale par provision, d'une attente qui se soutient de l'historicité eschatologique du projet kimbanguiste. En effet, le grand récit millénariste

2. Notre terrain s'étale de 1995 à aujourd'hui, dans la diaspora kimbanguiste de Belgique mais aussi en 19961997, en 2001 et en 2006 au Congo-Kinshasa (cf. Mélice 2006). De plus, nous avons fait effectuer des questionnaires à Kinshasa, notamment en 2007, par notre assistant de recherche (Fidèle kimbanguiste, il est aujourd'hui âgé d'une trentaine d'années, a fait des études supérieures non-universitaires et a un emploi).

3. L'EJCSK avance le chiffre officiel, mais non vérifiable, de dix-sept millions de fidèles kimbanguistes de par le monde. Elle estime généralement que les Kimbanguistes représenteraient environ 10\% de la population du Congo-Kinshasa, soit environ sept millions. Pour sa part, Asch, sur la base de recensions kimbanguistes effectuées en 1976, avance pour cette année le chiffre de 227.304 fidèles pour l'ensemble du Zaïre (Asch $1983: 326)$.

4. Les fidèles résident principalement dans les trois pays de l'expansion du kimbanguisme à l'époque coloniale, en l'occurrence le Congo-Kinshasa, le Congo-Brazzaville et l'Angola, mais aussi dans plusieurs pays d'Afrique (Zambie, Ruanda, Burundi, République centrafricaine, Kenya, Madagascar, Afrique du Sud, Nigéria, Cameroun, Gabon, Sénégal, Côte d'Ivoire), d’Europe (France, Angleterre, Belgique, PaysBas, Allemagne, Suisse, Espagne, Italie, Portugal, Finlande, Suède, Irlande), aux États-Unis, au Canada, au Brésil et en Australie.

5. Les ressources financières de l'EJCSK sont notamment issues de la pratique du nsinsani (collecte d'argent par compétition). En outre, l'EJCSK possède des écoles, des universités, des dispensaires, des hôpitaux, des centres agricoles, des logements, des temples, des centres administratifs, etc. 
qui supporte la doctrine renvoie à plus tard la réalisation de ce projet théologico-politique sans commune mesure avec la réalité politique actuelle.

\section{L'apolitisme de l'EJCSK}

L'apolitisme constitue un caractère essentiel de la doctrine kimbanguiste. Il est cependant manifeste qu'il n'est pas exclusif de positions idéologiques et, en particulier, de rejets idéologiques. Il est frappant qu'il est admis sans difficulté qu'à titre individuel, les Kimbanguistes adhèrent à des partis politiques. C'est là une constante qui se traduira encore lors des élections de 2006.

C'est dès 1957 que l'apolitisme du kimbanguisme est déclaré, dans « Mise au point sur le kimbanguisme » (CRISP, 1960 : 18-20). Le kimbanguisme vise à se dépouiller de l'apparence politique qui lui a valu d'être réprimé. Son apolitisme répond à une exigence de l'administration coloniale belge et conditionne la reconnaissance du mouvement par cette dernière. Dans le texte de la première Constitution de l'EJCSK, on peut lire : « L'E.J.C.S.K. se garde de toute tendance à caractère politique incompatible avec le rôle exclusivement spirituel qu'elle entend jouer au sein de l'humanité » (Luntadila 1990 : $27)^{6}$.

Au lendemain de l'Indépendance, le souci de l'apolitisme ira se confirmant. Dans les statuts de l'EJCSK du 5 mars 1960 (art.9, II, a), il est exigé des adeptes « de respecter les autorités (Épître de Paul aux Romains, $13: 1-3$ ). Que toute personne soit soumise aux autorités, car il n'y a pas d'autorité qui ne vienne de Dieu » (Asch 1983 : 129). Il est paradoxal que l'apolitisme kimbanguiste se concrétise dans ce qu'Asch désigne comme une conception de l'État en termes de « droit divin ». L'apolitisme kimbanguiste semble s'accommoder d'un fondement théologico-politique de l'État.

En 1962, le fils cadet de Kimbangu, Diangienda, chef spirituel de l'EJCSK de 1958 à 1992, attache l'EJCSK au principe, énoncé par le Christ : "Rendez à César ce qui est à César, et à Dieu ce qui est à Dieu ». De la sorte, il réitère " la démarcation entre le pouvoir spirituel et le pouvoir temporel ». Il contraint en outre les fidèles à « remplir toutes leurs obligations civiques ainsi que l'imposent les lois en vigueur dans les pays où ils ont élu domicile ». Mais, à nouveau, ce partage reste pris dans un horizon théologique. Diangienda précise en effet que l'EJCSK s'oppose « à toute idéologie, doctrine, ou théorie politique ou économique et sociale qui nie l'existence à Dieu » ou qui « tend à expliquer l'évolution historique du monde en écartant l'intervention divine », et il interdit aux fidèles kimbanguistes d'adhérer à ce type d'idéologie ou de doctrine (Martin 1981 : 141-144). Simplifiée à l'extrême, l'idéologie politique kimbanguiste se condense autour de plusieurs principes : le fondement théologico-politique du pouvoir, le rejet de l'athéisme, du marxisme et du darwinisme, l'apolitisme et la non-violence ${ }^{7}$. Toutefois, « la théologie kimbanguiste ne s'oppose pas à ce que les membres de l'Église, en leur qualité de citoyens, adhèrent à des partis politiques ou exercent des fonctions politiques » (Diangienda Kuntima 1984 : 285). Les Kimbanguistes attestent que jamais il ne leur fut

6. C'est ce texte constitutif qui sera déposé, en mars 1958, auprès du gouverneur général Cornelis, en vue d'obtenir la personnalité civile (Ustorf 1975 : 409).

7. Le principe kimbanguiste de la non-violence ne peut être négligé : cf. Diangienda 1984 : 73 et 206 ; Martin 1981 : 143 ; Mélice, à paraître ; Van Lierde 1986 : 19-31. 
imposé d'adhérer à un parti déterminé, ni interdit de manifester des préférences et des sensibilités politiques diverses.

\section{De la deuxième République à la Conférence nationale souveraine}

\subsection{L'EJCSK sous la deuxième République : entre contiguïté institutionnelle et oppositions individuelles}

C'est un fait incontestable que la position officielle de l'EJCSK à l'époque du mobutisme est celle d'une contiguïté institutionnelle. Il n'est cependant pas niable que de nombreux Kimbanguistes, dont des dignitaires, ont rejoint les rangs de l'opposition politique au régime sans qu'il leur en soit fait reproche de la part des dirigeants kimbanguistes.

En décembre 1971, Mobutu ne reconnaît plus que les seules Églises catholique, protestante et kimbanguiste (EJCSK) et fait dissoudre, puis interdire en 1972, les sectes. L'interdiction des sectes dissidentes kimbanguistes ${ }^{8}$ par Mobutu a permis à l'EJCSK de se renforcer institutionnellement et à Mobutu d'asseoir l'allégeance de l'EJCSK au MPR. Pour obtenir cette reconnaissance par l'État, il a fallu, selon Asch, que l'EJCSK se plie aux exigences de l'État zaïrois. Ces exigences, pense-t-elle, « influeront sur l'élaboration du kimbanguisme officiel » dont elle n'hésite pas à dire qu'il est « pro-mobutiste». C'est en termes d'idéologie qu'elle explique cette option : à l'époque, l'EJCSK naissante obéit à la contrainte d' « adapter le contenu idéologique du kimbanguisme à l'idéologie dominante de l'État, pour s'assurer la bienveillance des régimes successifs à l'égard de l'EJCSK dont, notamment celle du régime actuel [i.e. mobutiste] » (Asch 1983 : 101-102).

Il n'est pas nécessaire d'interroger longuement les affinités entre l'EJCSK et le régime mobutiste. André Droogers et Susan Asch ont traité cette question. Droogers avait déjà, en 1980, tracé une série de parallélismes entre Mobutu et Diangienda, entre l'État et l'Église kimbanguiste9. Pour sa part, Asch a conclu que 1'EJCSK a participé « activement à la diffusion des mots d'ordre mobutistes [comme le salongo, servir et ne pas se servir, etc.], à l'endoctrinement de ses adeptes et à la réalisation de la politique nationale [...]. D'Église en principe apolitique, le kimbanguisme officiel est aujourd'hui devenu support de l'idéologie de l'État, et un porte-parole du mobutisme ». Cette conclusion est excessive à nos yeux. Elle ne fait pas la part du principe kimbanguiste du respect des autorités. Il est vrai qu'Asch la nuance quelque peu. Elle distingue en effet entre le « kimbanguisme officiel » et ce qu'elle appelle le " kimbanguisme des Kimbanguistes » (Asch 1983 : 134-135) : «Le « kimbanguisme officiel» s'écarte du rôle traditionnel d'opposition politico-religieuse, tandis que le « kimbanguisme des Kimbanguistes» traditionalistes et dissidents représente sa prolongation historique à l'époque actuelle » (Asch 1983 :

8. Les premières dissidences, survenues au début des années 1960, furent bien souvent générées par des Kimbanguistes de renom comme, par exemple, Emmanuel Bamba, Simon-Pierre Mpadi ou Simon Kiamosi. Ces églises kimbanguistes dissidentes s'inscrivent régulièrement dans la dynamique des Églises de guérison étudiées par René Devisch (Devisch 1996, 1998 et 2000).

9. Tels que la manière commune d'organiser les visites de Mobutu et de Diangienda (voyage en C-130, inspection des gardes en uniforme, etc.), la pratique commune du salongo, l'appariement avec la campagne pour l'authenticité, etc. (Droogers 1980 : 204-205). 
292). Cette distinction qu'opère Asch entre deux espèces de kimbanguisme ${ }^{10}$ n'est pas dénuée de pertinence. Mais Asch ne se prononce pas sur la conception politico-religieuse des Kimbanguistes traditionalistes. De notre côté, bien que certains responsables kimbanguistes, dont Lucien Luntadila, le secrétaire général de l'Église, aient appartenu au MPR ${ }^{11}$, nous avons constaté qu'un certain nombre de dignitaires et de fidèles kimbanguistes furent, à titre personnel, en total désaccord avec le MPR, son idéologie et ses pratiques. Ainsi, on trouve parmi les Kimbanguistes une frange importante de Lumumbistes et de Tshisekedistes qui furent hostiles à Mobutu au prix, parfois, de l'exil. De même, E. Bena-Silu, le chef de Cabinet (depuis 1970) des chefs spirituels kimbanguistes était très proche de certains milieux politiques kongo ${ }^{12}$ opposés au MPR. À ce propos, nous avons observé depuis les années 1990, qu'en dépit des déclarations de principe supraethniques et universalistes de l'EJCSK, une part non négligeable, bien que quantitativement incernable, d'ethnonationalistes kongo sont présents au sein de l'Église. Ceux-ci aspirent à la restauration de l'ancien royaume de kongo, mais davantage sous la forme atténuée d'un renforcement du lien politique entre les trois pays de l'aire kongo : les deux Congo et l'Angola. Toutefois, bien que se dissociant radicalement du mouvement du Bundu dia Kongo ${ }^{13}$, ces ethnonationalistes s'inspirent parfois de certaines croyances ${ }^{14}$ de celui-ci, lesquelles supportent ses thèses confédéralistes ${ }^{15}$. Nous ne pouvons nous étendre sur ce point ici. Ce phénomène, sans doute déjà présent sous le mobutisme, semble s'être

10. Asch voit dans le « kimbanguisme des Kimbanguistes » traditionalistes, un kimbanguisme opposé au réformisme de l'EJCSK. Elle insiste abondamment sur l'opposition entre le kimbangucentrisme (qui identifie Kimbangu à l'Esprit Saint) des traditionalistes et le christocentrisme de 1'EJCSK réformiste, c'està-dire rattachée au COE. Elle évoque, sans la développer, la part d'opposition politico-religieuse des fidèles traditionalistes. Asch estime que ceux-ci sont majoritaires en nombre au sein l'EJCSK. C'est pourquoi l'EJCSK a toujours opté pour une politique de tolérance à l'égard des croyances traditionalistes (Asch 1983 : 139-179, 292-293).

11. Lucien Luntadila fut membre du Comité Central du MPR et député du Haut Conseil de la République. Par ailleurs, on reproche souvent à Charles Kisolokele, le fils aîné de Kimbangu, d'avoir été député et secrétaire d'État sous Mobutu. En réalité, il ne conserva son poste de député que jusqu'en 1966. C'est l'un de ses fils qui aurait rempli des mandats politiques (de secrétaire d'État) sous Mobutu.

12. Il nous a été rapporté à plusieurs reprises que Bena-Silu est très proche de certains « milieux politiques kongo ». Dans le passé, il aurait fréquenté, au Congo-Brazzaville, les opposants kongo au régime du Président Marien Ngouabi. Et depuis l'assassinat de ce dernier, en 1977, il serait interdit de séjour au Congo-Brazzaville.

13. Parce que celui-ci est violent et aussi parce qu'il est anti-chrétien.

14. Notamment les croyances supportées par la symbolique de la marmite traditionnelle kongo dont les trois pieds renvoient aux trois clans kongo initiaux.

15. Le Bundu dia Kongo (BDK), fondé en 1986, est un mouvement ethnonationaliste jugé subversif par l'État zaïrois, puis congolais, parce que revendiquant une confédération kongo. Kimbangu y occupe la place d'un prophète. Sur le BDK, cf. son périodique Kongo Dieto ; Ne Muanda Nsemi, 1991, 1993, 1995 et 1998 ; Bernhard, 1999. 
accru dans les années 1990, à la faveur de la transition politique ${ }^{16}$ ainsi que du décès de Diangienda et des forces centrifuges que ce décès suscita.

C'est dire qu'il y avait déjà sous le mobutisme une pluralité de préférences politiques parmi les fidèles kimbanguistes. Cette pluralité de sensibilités politiques constitue probablement l'un des motifs du refus de l'EJCSK de devenir la religion officielle de l'État zaïrois comme ce dernier le lui a proposé dans la seconde moitié des années soixante ${ }^{17}$.

\title{
2.2. La Conférence nationale souveraine (CNS) et l'approche de la " troisième
} République " : la défiance à l'égard du processus de démocratisation

Lors de la tenue de la CNS, l'EJCSK témoigne à l'égard de la référence à la démocratie, d'une suspicion qui s'alimente à son projet théologico-politique. À nouveau, il est incontestable que cette prise de position officielle n'a pas empêché nombreux de ses membres de conserver des sensibilités politiques divergentes.

En 1996, Luntadila, alors secrétaire général de l'Église, rapporte qu'à l'époque de la Conférence nationale souveraine, Diangienda prononça la parabole suivante :

\begin{abstract}
Vous connaissez la grenouille et ses yeux, ils sont gros. Un jour le mâle et la femelle ont eu une petite grenouille. Ils ont vu qu'elle a de gros yeux. Ils se sont plaints, croyant qu'elle avait une maladie. Ils sont allés chez un guérisseur et lui ont demandé ce qu'ils devaient faire avec la petite malade. Le guérisseur va prendre un miroir et leur demander de fermer les yeux et de les rouvrir quand il aura tapé dans les mains. Il a mis le miroir en face d'eux. Il leur a demandé de regarder. Les deux grenouilles ont vu qu'elles avaient elles aussi les yeux "sortis 》 comme la petite grenouille.
\end{abstract}

Luntadila ajoute : «Alors Diangienda disait : «Vous êtes tous les mêmes. Vous critiquez ceux qui ont détourné des fonds lors de la deuxième République, mais l'opposition en fait partie. Alors vous avez tous de gros yeux. Vous êtes tous malades. Ce n'était pas ça la solution »(Luntadila, 1996, Kinshasa).

L'EJCSK fut néanmoins représentée à la CNS par une trentaine de Kimbanguistes. Un Kimbanguiste déplore l'attitude des conférenciers de la CNS qui accablèrent de leurs huées le député kimbanguiste Likutu quand celui-ci intervint pour demander que l'on reconnaisse Kimbangu en tant que libérateur. Diangienda réagit alors devant les Kimbanguistes en ces termes : « Comme ils ont ridiculisé mon père, je vous dis que cette Conférence nationale n'aboutira à rien. Ces papiers-là des résolutions de la CNS, vous

16. Lors de la première transition (avril 1990-mai 1997), le Bas-Zaïre est marqué par « une résurgence d'un nationalisme Kongo nourri de l'utopie d'une restauration de l'ancien royaume du même nom » (de Villers 1998 : 93), notamment de la part du mouvement politico-militaire et religieux, le Royaume Uni du Congo, du « roi Nsemi Minzele [ou Misele] ». Mais « l'existence de cette organisation revêt une signification plus symbolique que politique » du fait de «l'émiettement prononcé du leadership Kongo » (de Villers 1998 : 93). cf. aussi De Boeck et Plissart 2005 : 103.

17. Cette proposition, dont elle reconnaît l'effectivité, Marie-Louise Martin (protestante et ancienne doyenne de la Faculté de théologie kimbanguiste) l'explique par l'admiration que plusieurs politiciens zaïrois vouaient à Kimbangu, en qui ils reconnaissaient un héros national. Elle commente comme suit le motif du refus : « l'Église ne saurait être dépendante de l'État : elle doit se bâtir exclusivement sur Jésus-Christ » (Martin 1981 : 145). Cette explication rencontre l'opinion des Kimbanguistes qui motivent ce refus par le principe d'apolitisme de l'EJSCK. 
les retrouverez plus tard sur les marchés chez les vendeurs d'arachides qui les utiliseront comme emballages » (OO, avril 2006 et ZZZ, mai 2006, Kinshasa) ${ }^{18}$.

En 1997, un fidèle kimbanguiste fit part des propos de Diangienda, teintés de millénarisme, sur la CNS et sur la « troisième République » :

Sur la Conférence nationale souveraine, Papa Diangienda a dit : " Elle va échouer parce que tant que vous ne connaîtrez pas mon père Simon Kimbangu, des élections, il n'y en aura même pas». D'où il disait : "Nous allons nous arrêter à un moment donné et tout va recommencer à zéro avec un gouvernement de Dieu». [...] Il disait : "La troisième République, c'est moi». La démocratisation, ce n'est qu'une mascarade. (JJ, janvier 1997, Kinshasa $)^{19}$

En effet, on nous dit à maintes reprises que Diangienda désignait la démocratie comme « l'ordre du démon ». " La démocratie, commente un Kimbanguiste, ne fait qu'opposer les gens. Le pouvoir vient de Dieu ; le pouvoir ne vient pas des hommes. Le projet politique de Dieu n'est pas la démocratie. C'est un roi souverain éternel, c'est Dieu » (K, décembre 1996, Kinshasa) ${ }^{20}$. Un théologien kimbanguiste nous a livré une autre déclaration qu'aurait faite Diangienda sur la troisième République :

Il nous a dit : "Nous suivons le rythme trinitaire : Dieu le Père, le Fils et le SaintEsprit. Nous attendons la troisième République qui sera celle du Saint-Esprit, du Molimo Santu. Ce sera sous la direction de Simon Kimbangu». Dans l'Église kimbanguiste, Papa Simon Kimbangu, nous le considérons comme le Consolateur promis par Christ [le Saint-Esprit]. L'Église kimbanguiste insiste sur le fait que tant qu'on ne reconnaîtra pas Simon Kimbangu, rien ne marchera. Au Congo, qu'on le veuille ou non, Kimbangu est capable, par la personne du Christ, de guider les hommes politiques. (D, septembre 2001, Kinshasa) $)^{21}$

C'est à l'Église kimbanguiste qu'il devrait revenir d'apporter la solution aux problèmes politiques : «Avant la Conférence nationale souveraine, Papa Diangienda avait dit que ce serait inutile de perdre beaucoup de temps dans ce genre de rencontre politique, mais que la vraie solution proviendrait de la purification de nos cœurs. C'est ainsi qu'il a répété notre équation : amour-commandements de Dieu-bonnes œuvres ${ }^{22}$. $($ (L, décembre 1996, Bas-Zaïre, Kwilu-Ngongo) ${ }^{23}$.

18. OO est un fidèle kimbanguiste, est âgé aujourd'hui d'une trentaine d'années, a fait des études secondaires supérieures et est sans emploi. ZZZ est un dignitaire administratif de l'Église, est âgé d'une cinquantaine d'années, a fait des études universitaires et occupe un emploi dans l'Église.

19. JJ est un fidèle de l'Église, âgé d'une quarantaine d'années, a fait des études supérieures non universitaires et est sans emploi.

20. K est un dignitaire administratif kimbanguiste, est aujourd'hui âgé d'une soixantaine d'années, a fait des études universitaires et a un emploi.

21. D est un dignitaire ecclésiastique kimbanguiste, est âgé d'une cinquantaine d'années, a fait des études universitaires et a un emploi.

22. Cette «trilogie kimbanguiste », correspond à l'« éthique » kimbanguiste et est considérée comme le « testament» laissé par Diangienda.

23. L est un dignitaire ecclésiastique kimbanguiste, est âgé d'une cinquantaine d'années, a fait des études universitaires et a un emploi. 
En 1996, un fidèle kimbanguiste rapporte des propos tenus par Diangienda sur le pouvoir de transformation propre aux Kimbanguistes : «Diangienda a dit : "Vous Kimbanguistes, vous avez un rôle important à jouer dans cette histoire de l'humanité. En 1956, les prières de nos papas, de nos grands-pères, on a vu ce que ça a apporté pour le changement. Je pense que c'est le temps». » (G, mars 1996, Bruxelles $)^{24}$. L'allusion à 1956, au « bolingo ya 1956 », au « réveil de 1956 », à cet « âge d'or » du kimbanguisme sous sa forme communautaire (le $x$ ) n'est pas insignifiante. C'est l'époque de l'« action ouverte », laquelle a mené à la reconnaissance, en 1959, du kimbanguisme. Cette allusion n'est pas sans suggérer que tout changement, y compris politique, puisse être déterminé par le kimbanguisme.

Ces quelques passages d'entretiens soulignent combien l'EJCSK a conçu et développé, particulièrement depuis les années 1990, un projet théocratique, en résonance avec la dimension millénariste qui lui est consubstantielle (cf. Mélice 2001). Durant cette période, l'hostilité à l'égard du processus de démocratisation du pays était assez généralisée au sein de l'EJCSK. Mais cette animosité n'a pas empêché que de nombreux Kimbanguistes adhèrent à divers partis et témoignent de sensibilités politiques divergentes. Par son pluralisme déclaré, l'EJCSK a toujours autorisé ces engagements.

Le décès de Diangienda survint en juillet 1992. Une foule de Kinois lancèrent sur son convoi funéraire des projectiles divers en proférant des injures telles que « hibou », « traître », etc. (Manny 1992 : 3), " magicien », " franc-maçon » (VV, 2007, Kinshasa, questionnaire soumis par C $)^{25}$. Le substantif « hibou » est le surnom qui fut donné par la population zaïroise aux Forces d'Intervention Spéciales zaïroises (FIS) ${ }^{26}$. Cette référence aux FIS, aux « Hiboux », est elle-même supportée par la signification de sorcellerie qui lui est traditionnellement conférée. Diangienda faisait l'objet d'accusations de sorcellerie depuis longtemps ${ }^{27}$. Il était soupçonné de puiser son pouvoir aux « forces de l'invisible », en d'autres termes de pratiquer la «politique du ventre » (Bayart $1989: 12$ ).

L'hostilité de la population à l'égard de Diangienda au moment de son décès a été accrue par le contexte historique. À ce moment-là, se réunissait la CNS, qui fut l'occasion de contester le régime mobutiste et ses affidés. Ce fut l'époque de ce qu'on a baptisé le « déballage $»^{28}$. En outre, la méfiance que la population ressentait à l'égard

24. G est un fidèle kimbanguiste, est aujourd'hui âgé d'une cinquantaine d'années, a fait des études supérieures non-universitaires et est sans emploi.

25. VV est un dignitaire administratif kimbanguiste, est âgé d'une soixantaine d'années, a fait des études secondaires supérieures et occupe un emploi dans l'Église.

26. Dans les années 1990, celles-ci, fondées par Honoré Ngbanda (le « Terminator ») terrorisèrent, de jour et surtout de nuit, la population zaïroise et les opposants politiques. "Les gens avaient donné à Papa Diangienda le nom de « hibou» parce qu'on disait qu'il était l'agent de Mobutu. Les « hiboux» de Ngbanda, c'était l'injure !» (Q, avril 2007, Liège : Q est un dignitaire administratif kimbanguiste, est âgé d'une septantaine d'années, a fait des études secondaires moyennes et est retraité ).

27. Ainsi, les fréquents accidents mortels sur la route de l'ex-Bas-Zaïre, ainsi que le naufrage du bateau Sakaroni et l'écroulement du pont au beach Ngobila, furent très tôt attribués par la population zaïroise au pouvoir « sorcier », « mystique » de Diangienda.

28. À ce déballage appartiennent les « révélations » faites par Sakombi et par Bofossa (cf. Ndaywel 1993 : 33-50). À ce « déballage » ressortit l'accusation de proximité entre l'EJCSK et le régime mobutiste, et plus particulièrement entre Joseph Diangienda et Joseph Mobutu, les « deux Joseph ». 
des Kimbanguistes avait été accentuée récemment par leur refus de participer $^{29}$ à la marche pacifique (dite la « marche de l'espoir ») $)^{30}$ que les Chrétiens avaient organisée, le 16 février 1992 pour protester contre la suspension de la CNS.

Le 12 juillet 1992, le Président Mobutu vint se recueillir devant la dépouille de Diangienda. Dans son discours, il invoqua un événement qui se serait produit en 1958 : Diangienda l'aurait béni et l'aurait, par anticipation, investi de son pouvoir à venir. Cette bénédiction de Mobutu a longtemps assis, aux yeux de nombreux Kimbanguistes, la légitimité de son pouvoir, qui, du coup, leur semblait émaner de Dieu. Le discours de Mobutu accrut dans la population le sentiment d'une proximité excessive entre les deux hommes. Mais il fut aussi compris comme un essai de récupération politique du décès de Diangienda. Mobutu, dont le pouvoir était alors contesté, tentait ainsi, bien entendu, de réaffirmer sa légitimité en invoquant une référence religieuse prophétique.

\section{L'EJCSK, les Kabila et les élections de 2006}

L'EJCSK a donc représenté et représente jusqu'à ce jour une force politiquement non négligeable. Il est d'autant plus frappant que ce poids effectif se soit autorisé d'une déclaration jamais démentie de neutralisme qui s'accommode en fait du pouvoir établi. Suivant son principe de respect des autorités, l'EJCSK avait reconnu le pouvoir de Mobutu. Elle allait de même reconnaître ceux de Laurent puis de Joseph Kabila. L'incompatibilité foncière de la doctrine kimbanguiste avec les principes démocratiques n'empêchera pas que de nombreux Kimbanguistes - dont le chef spirituel lui-même - s'impliquent dans les élections en RDC en 2006. C'est ce constat d'une apparente inconséquence que nous chercherons à élucider en conclusion.

Laurent Désiré Kabila « répétait souvent que Simon Kimbangu est un des pionniers de la politique congolaise »(Q, juillet 2007, Liège $)^{31}$. Dans la lignée politique directe de Kimbangu, L.D. Kabila inscrivait Lumumba. C'est au Kimbangu nationaliste ou encore patriote qu'il rendait hommage. Il semble cependant que L.D. Kabila se fit plus discret que son prédécesseur et que son successeur dans ses rapports avec l'EJCSK, comme l'atteste un Kimbanguiste : « Kabila père n'avait pas tellement de relations avec l'EJCSK car, à aucun instant, il n'a visité l'Église ni son chef; bref, il ne s'intéressait pas du tout à l'EJCSK » (C, janvier 2007, Kinshasa, par téléphone $)^{32}$.

Joseph Kabila réitéra les propos de son père sur le nationalisme et le patriotisme de Kimbangu. Dans son discours d'investiture au Palais du Peuple, le 28 janvier 2001, « il n’a pas parlé des Kimbanguistes mais du moins il a encouragé la démarche de révolution entreprise par Simon Kimbangu » (C, janvier 2007, Kinshasa, par téléphone). Dans un message adressé à l’Église en 2006, Joseph Kabila déclara : «L'Église kimbanguiste, c'est notre Église patriotique parce que son fondateur est le premier de nos patriotes » (EJCSK 2007 : 442). Davantage que son père, Joseph Kabila s'est montré sensible à

29. C'est Diangienda qui avait donné l'instruction de ne pas participer. Cette décision, les Kimbanguistes la justifient par le principe kimbanguiste de respect des autorités.

30. Cf. de Villers, $1995: 230$; Malenge, $1992: 120-123$; Ndaywel è Nziem, $1998: 777$.

31. Q est un dignitaire administratif kimbanguiste, est âgé d'une septantaine d'années, a fait des études secondaires moyennes et est retraité.

32. C est un fidèle kimbanguiste, est aujourd'hui âgé d'une trentaine d'années, a fait des études supérieures non universitaires et a un emploi. 
l'importance politique de l'Église. Il a rendu des visites officielles aux chefs spirituels Salomon Dialungana puis Simon Kimbangu Kiangani et les a reçus en audience. Le 17 mai 2001, date anniversaire de l'arrivée de L.D. Kabila à Kinshasa, c'est au centre d'accueil kimbanguiste de Kinshasa (dans la commune de Kasa-Vubu) que s'est tenue une cérémonie politique « organisée par l'État et à laquelle Joseph Kabila et son gouvernement avaient pris part. C'est lors de cette cérémonie que Joseph Kabila avait libéralisé les activités des partis politiques et parlé de démocratie en RDC » (QQ, avril 2007, Kinshasa, questionnaire soumis par C) ${ }^{33}$. On peut supputer que cet événement fut l'occasion de conforter la conviction kimbanguiste que la reconnaissance de Simon Kimbangu constituait le préalable obligé vers l'établissement d'une troisième République.

Nous nous trouvions à Kinshasa durant la période pré-électorale, en avril et en mai 2006. Une trentaine de Kimbanguistes étaient candidats à la députation. Ils appartenaient à divers partis politiques ou se présentaient comme candidats indépendants. Il semble que leurs candidatures aient été particulièrement instiguées par Likutu, lequel fut l'un des rares députés kimbanguistes sous la seconde République (MPR). Le 9 mai 2006, au cours d'une émission télévisée, nous avons entendu Likutu inciter les Kimbanguistes à « cesser de pratiquer la politique de la chaise vide ». Il mettait en évidence les impasses auxquelles peut conduire une certaine interprétation de l'apolitisme : « Les Kimbanguistes se plaignent de ne pas avoir de représentants au pouvoir, alors que les Kimbanguistes refusent de s'impliquer dans la politique. C'est pourquoi, il y a quelques mois, Likutu a incité à ce que les Kimbanguistes se présentent comme candidats députés. » $(C$, avril 2006, Kinshasa).

Dans le contexte électoral, les marques de respect qu'échangent le chef de l'Église et le chef de l'État ne sont évidemment pas exemptes de stratégie : « Kabila est très attentif à Simon Kimbangu Kiangani [chef spirituel], surtout qu'il attend son soutien même tacite pour avoir les Kimbanguistes avec lui »(C, mai 2006, Kinshasa). Il est vrai que Joseph Kabila a invoqué la figure, emblématique et charismatique aux yeux de nombreux Congolais kimbanguistes, mais aussi non-kimbanguistes, du prophète Simon Kimbangu. En janvier 2006, quelques mois avant les élections, paraissait sur le site web officiel du Président de la République, un article émanant du bureau de son porte-parole, intitulé « Le destin exceptionnel de Joseph Kabila ». Cet article se réclamait d'une prophétie de Simon Kimbangu et visait à conférer une dimension providentielle aux destinées politiques de la RDC et, particulièrement, à celle de Joseph Kabila. L'auteur de l'article, Marcel Nzazi Mabidi, citait et commentait la prophétie de Kimbangu en ces termes :

Ce qu'a prophétisé Simon Kimbangu. Avant son arrestation en 1921, Simon Kimbangu prophétisant sur l'avenir du Congo belge a dit :

Le Congo sera indépendant un jour. Pendant 40 ans, le pays sera mis à feu et à sang et connaîtra d'énormes difficultés et souffrances de tous genres, puis viendra le bonheur. Le pays sera d'abord dirigé par un mouton. Cet homme sera originaire de la province où je suis né. Le pays sera ensuite dirigé par un fauve qui viendra écarter le mouton. Pendant le règne du fauve marqué par la terreur, le pays sera saccagé. Il manquera d'argent dans le pays. Même les banques seront vides. Viendra ensuite un homme, un météore, originaire de la province où je finirai ma vie [Katanga]. Son règne sera très court. Son principal rôle sera de chasser le fauve du pouvoir.

33. QQ est un dignitaire administratif de l'Église, est aujourd'hui âgé d'une quarantaine d'années, a fait des études universitaires et occupe un emploi dans l’Église. 
Puis viendra quelqu'un, un jeune homme sage. C'est lui qui sauvera ce pays et apportera au peuple le bonheur, la vraie indépendance.

L'auteur de l'article ajoute immédiatement : «Et la prophétie de Kimbangu s'est réalisée ». Il traduit et explicite abondamment la signification de cette prophétie : l'Indépendance en 1960 ; le «mouton »: Kasa-Vubu ; le «fauve »: Mobutu ; le « météore » : L.D. Kabila et, enfin, le « jeune homme sage », à propos duquel l'auteur pose la question, sans doute rhétorique : « ne serait-il pas Joseph Kabila qui préside aux destinées de la RDC ? » (Nzazi Mabidi 2006). Un journaliste de Jeune Afrique, François Jourdan, commente le recours à cette "référence mythique (et mystique) pour des millions de Congolais » : "En termes de légitimation d'un pouvoir venu de nulle part ou presque (« Il m’est tombé dessus », a confié un jour l'intéressé), le gain n'est pas négligeable - à condition d'y croire bien sûr » (Jourdan 2006). L'allusion aux rumeurs sur les origines indécises de Joseph Kabila, sur sa filiation et sur sa nationalité, est transparente : l'auteur de l'article suggère que, quoi qu'il en soit, c'est d'une tout autre origine, d'une origine radicalement théologique que Joseph Kabila recevrait ainsi sa légitimité. Ajoutons qu'en outre Joseph Kabila pouvait toucher de la sorte un électorat qui lui restait très peu acquis, celui de la Province du Bas-Congo (aujourd'hui Province du Kongo central), en s'autorisant de la figure emblématique du grand prophète kongo que représente Kimbangu.

Cette prophétie de Kimbangu relative aux futurs chefs d'État, la propagande kabiliste ne l'a pas inventée. Elle est connue des divers milieux kimbanguistes. Diverses versions écrites de cette prophétie circulent, toutes inspirées de la version, traduite du kikongo en français par Passi Mpiosso et publiée en 1998 dans l'hebdomadaire La Renaissance (voir Passi Mpiosso 1998). Cette prophétie, Kimbangu l'aurait énoncée le 17 juin 1944 dans la prison d'Élisabethville devant l'un des rares visiteurs congolais autorisés à le rencontrer, Jean Kiansumba. Une des versions de la prophétie (version explicitement reprise de la version publiée dans La Renaissance et très probablement issue d'une Église de guérison, celle du prophète $\mathrm{Ntau}^{34}$ ), vendue à la porte du centre d'accueil kimbanguiste de la commune de Kasa-Vubu, décrit, en les résumant, les figures et les règnes des « quatre chefs d'État » prophétisés par Kimbangu : le premier chef d’État « sera un homme digne, modèle, exemplaire et responsable [qui] finira empoisonné »; le deuxième chef d'État « sera un grand voleur, destructeur, et détourneur des biens publics [...]; le troisième chef d'État « ne fera pas longtemps au pouvoir. Sa mauvaise politique divisera le pays en plusieurs parties (ou foyers de tensions). [...] Lui aussi, comme le second, sera rejeté par le peuple et chassé du pouvoir »; le quatrième chef d'État, enfin, sera un « homme très calme et discipliné [...] Pendant son règne, le Congo sera prospère. Il n'y aura plus d'esclavage » (voir Passi Mpiosso 1998). Les versions écrites de cette prophétie, publiées par des Kimbanguistes de l'EJCSK, l'une sur deux sites web de la diaspora kimbanguiste (et qui fait référence à la publication de la prophétie dans La Renaissance), l'autre dans le livre du Kimbanguiste Bandzouzi (qui ne cite jamais aucune source), ne sont pas tout à fait identiques. Elles ont en commun de décrire les trois premiers « présidents » ou « chefs » dans des termes assez semblables à la version

34. Jaak Le Roy a étudié l'Église de guérison du prophète Ntau à Selembao (Kinshasa). Cf. Le travail thérapeutique dans les Églises de guérison à Kinshasa. Restructuration de la personne et du réseau familial, dans $<$ http :/www.psychmedia.it /neuro-amp/98-99-sem/leroy-fr.htm> 
que nous venons de citer ; mais elles se séparent sur le point, ici crucial, de l'annonce claire d'un quatrième « Président» ou " chef ». La version de Bandzouzi dit que le « troisième chef $[\ldots]$ partira vite du pouvoir. Lorsque vous obtiendrez l'Indépendance, gardez-vous de vous livrer au pillage car le maître de ce monde sera le maître de la terre et des hommes " (Bandzouzi 2002 : 204). La version figurant sur les sites web kimbanguistes dit : «Le troisième Président [...] vous le tuerez. Quand vous serez libérés, abstenez-vous du vol car le responsable du pays, à ce moment-là, sera un chef de terre et du peuple » (Élisabethville). Confrontées aux autres versions, ces deux versions laissent peser quelque équivoque sur la quatrième figure qu'elles nimbent d'une connotation plus millénariste. D'après nos informations, une majorité de Kimbanguistes de l'EJCSK se réfèrent à cette version de la prophétie de Mbanza-Nsanda, qui annonce trois « chefs » suivis d'un « chef » aux traits incertains. Nous le verrons plus loin, l'Église kimbanguiste nourrissait l'attente d'un « homme providentiel» dont les dimensions eschatologique et politique s'entremêlaient confusément à la veille des élections. Pour mesurer la portée de cette prophétie de Kimbangu aux yeux des Kimbanguistes, il faut rappeler qu'à la croyance eschatologique kimbanguiste, selon laquelle la " troisième République » coïncide avec le moment de la Fin, s'articule une croyance eschatologique relative à la « quatrième génération ». En un mot, la quatrième génération et la troisième République se superposent : l'une comme l'autre correspondraient au règne du Saint-Esprit. Il était d'ailleurs fréquent, avant les élections, d'entendre les Kimbanguistes dire que le fait qu'il y ait trois Républiques et quatre Présidences de la République congolaise (comme les quatre générations) constituait un signe eschatologique.

Quant à l'attitude bienveillante dont témoigne, de son côté, le chef de l'Église kimbanguiste à l'égard de Kabila, elle semblerait dictée par le principe kimbanguiste du respect des autorités en place et de l'obéissance à leur égard. Sur ce point, un fidèle kimbanguiste candidat aux élections livre ses impressions : "Quand plusieurs d'entre nous qui sommes candidats, sommes allés à Nkamba demander l'autorisation au chef de nous présenter comme députés aux élections, le chef a dit d'accord. Mais il a donné l'ordre qu'aucun Kimbanguiste ne se présente comme candidat au poste de Président. Tout le monde a compris que c'est parce qu'un candidat président, il en soutient déjà un, c'est Kabila. Le chef de l'Église favorise le chef de l'État. Il ne le dit pas ouvertement mais ça se sait. Cela va certainement influencer le vote des Kimbanguistes pour Kabila » (C, Kinshasa, mai 2006) $)^{35}$.

Mais, à côté de l'implication kimbanguiste dans le processus électoral, un discours peu favorable aux élections et à la démocratie en général se faisait également entendre, réactivant, en somme, le discours dominant durant les années 1990.

Du 5 au 9 juin 2005, s'est tenu à Nkamba un « séminaire-atelier de concertation entre l'Église kimbanguiste et la Commission électorale indépendante » (Mambu-ma-Mwana 2005 : 5), au terme duquel l'EJCSK s'est engagée à contribuer, sur le plan national, au « programme de sensibilisation et d'éducation civique et électorale » et à mettre à la disposition de la Commission électorale indépendante (CEI) ses infrastructures. Lors de la cérémonie d'ouverture de ce séminaire, le chef de l’Église aurait souligné que « les élections qui seront bientôt organisées dans notre pays symboliseront la libération du

35. C est un fidèle kimbanguiste, est aujourd'hui âgé d'une trentaine d'années, a fait des études supérieures non universitaires et a un emploi. 
peuple congolais comme l'avait prédit l'Envoyé Spécial du Christ, Papa Simon Kimbangu en 1921 » (Mambu-ma-Mwana 2005 : 5). Les propos du chef, parce qu'ils sont relatifs à la prophétie de Kimbangu analysée précédemment, comportent quelque équivoque et possèdent une allure millénariste. En juillet 2005, le chef aurait tenu les propos suivants à ses fidèles, rapportés par le journaliste kimbanguiste qui signe de ses initiales AMM : «La République démocratique du Congo a une lourde charge quant au patrimoine spirituel dont elle regorge. Certes, il arrivera un moment où le monde cherchera où se trouve Dieu. En ce moment-là précis, la République Démocratique du Congo accueillera toutes les nations du monde ». Et, évoquant " la situation socio-économique et politique », il aurait ajouté : « Nous attendons l'Indépendance spirituelle. Nos Papas [les trois fils de Kimbangu] ont tout fait, nous n'attendons que l'accomplissement des promesses » (AMM, $1^{\text {er }}$ août $2005: 3$ ).

Néanmoins, en dépit de ces réserves de principe, le chef spirituel s'est enrôlé, le 14 août 2005, sur la liste des électeurs. Il aurait invité, rapporte un journaliste du Kimbanguisme Info, « pour la énième fois, les fidèles Kimbanguistes en âge de voter à s'inscrire sur la liste des électeurs. Car voter, c'est un devoir civique ». Comme pour justifier son intervention, il aurait alors invoqué le respect tout aussi principiel des pouvoirs établis, et rappelé « qu'obéir à l'État, aux lois du pays ou encore aux autorités établies, est l'un des préceptes kimbanguistes. La Bible ne dit-elle pas qu'il faut rendre à César ce qui est à César et à Dieu ce qui est à Dieu ? "(AMM, 26 septembre $2005: 2$ ). Il n'est dès lors pas surprenant qu'à propos du référendum constitutionnel de décembre 2005, un journaliste kimbanguiste, faisant état de l'attitude du chef spirituel, la commente en ces termes :

\begin{abstract}
Respectueux des lois du pays, son Éminence Simon Kimbangu Kiangani [...] est allé, le dimanche 18 décembre 2005, au bureau de vote du référendum s'acquitter de son devoir civique. Loin de nous l'idée de savoir a-t-il voté pour le OUI ou pour le NON, il sied de noter que le $n^{\circ} 1$ de l'Église kimbanguiste a voté pour le bonheur du peuple congolais et de l'humanité tout entière. [...] En tout état de cause, S.E. Simon Kimbangu Kiangani ne mâche pas ses mots pour dire que la paix et le bonheur des Congolais ne viendront pas d'ailleurs sinon de Nkamba Nouvelle Jérusalem [ville sainte des Kimbanguistes]. [...] À notre avis, seul Dieu est capable de donner la paix en RDC selon les Écritures divines. (Psaumes $127: 1-2)^{36}$ (LKI $2005: 3)$
\end{abstract}

Les réserves à l'égard des élections peuvent être illustrées par les propos suivants tenus par un vieux dignitaire kimbanguiste :

Un chrétien, c'est un citoyen, c'est un électeur. Nous, on nous enseigne que nous devons respecter l'autorité. Elle nous dit de passer par les élections. [...] Maintenant, ce que va dicter l'Esprit dans tout ça, nul ne le sait; puisque finalement, c'est Dieu qui dirige tout ça. La pensée des hommes n'est pas celle de Dieu. Des fois, les Kimbanguistes sont sceptiques dans cette affaire-là de démocratie électorale. Démocratie, ça peut exister, mais électorale, c'est autre chose, parce que ça cache beaucoup de choses... Tout ce que je peux vous dire, c'est que nous nous acheminons vers un moment où Dieu doit instaurer son règne. Ainsi que Simon Kimbangu l'avait prédit en 1921, à Mbanza Nsanda, avant qu'il ne se fasse arrêter : "Dans les temps qui viendront, il ne restera qu'un seul pouvoir, une seule

36. Psaumes $127: 1$ : « Si l’Éternel ne bâtit la maison. Ceux qui la bâtissent travaillent en vain. Si l'Éternel ne garde la ville. Celui qui la garde veille en vain ». 
langue, une seule Église, et c'est moi, Simon Kimbangu, qui le ferai marcher». Si nous entrons dans cette ère-là, de l'établissement de l'ordre divin, à quoi servira encore la démocratie? Puisque Dieu est omniscient et omnipotent, qu'il connaît tout, avec qui va-t-il discuter? Tout comme il peut permettre les élections, puisque c'est un Dieu compatissant. Il veut voir comment les hommes vont faire. Mais il sait comment ça va se terminer. (U, mai 2006, Kinshasa) ${ }^{37}$

Dans la même perspective, sur un site kimbanguiste ${ }^{38}$, Basile Akiele, qui est le pasteur national des États-Unis, dévoile des positions théologico-politiques, anti-démocratiques et rétives au principe électoral :

L'approche de gouvernance divine n'est pas au bout des urnes. [...] Les positionnements démocratiques sont incomplets et imparfaits aux yeux de l'Éternel. La démocratie est une fabrication basée sur des considérations humaines. Ce n'est pas un concept divin. [...] Le vote est humain et n'est pas une valeur absolue, car il est hasardeux, imparfait, incomplet et souvent discutable, porteur de troubles, de haine et de discorde. [...] Par contre, ce que Dieu veut (sans campagne ni vote) est magnifique, forcément merveilleux [...] Tout pouvoir venant de Dieu, l'idéal serait de laisser l'Éternel faire lui-même le choix des ses élus qu'il révélerait suivant son programme. (Akiele)

La tension entre deux formes de discours sur les élections est récurrente. Les deux exemples suivants en sont de bonnes illustrations. D'abord, un candidat à la députation a rappelé la prophétie de Diangienda selon laquelle « il n’y aura pas d'élections dans ce pays ». En fait, précisa-t-il, " nous les Kimbanguistes, nous attendons un homme providentiel. La fin du monde, c'est pour bientôt» (C, mai 2006, Kinshasa). Ensuite, une émission kimbanguiste, "L'Église en marche » recevait fréquemment les candidats aux élections. Or, le directeur de la chaîne kimbanguiste, Mambu Mamwana, procéda, dans une allocution aux allures de prédication (lors de l'émission du 29 avril 2006), à une mise en garde contre « les politiciens qui veulent flatter l’Église kimbanguiste pour les élections. Il n'y a que Papa Simon Kimbangu qui mettra la paix. Tant qu'on ne reconnaîtra pas Simon Kimbangu, le monde ne sera pas en paix. Il faut d'abord reconnaître Simon Kimbangu comme Sauveur, comme Envoyé de Dieu ! ». Du reste, le chef spirituel luimême aurait rendu le processus électoral tributaire de la reconnaissance préalable de Kimbangu. Il aurait en effet prophétisé, nous rapporte un fidèle kimbanguiste, que « tant que tout ce monde-là continuera à ignorer notre père Simon Kimbangu, qui a beaucoup souffert pour ce pays, ce pays ne décollera pas » (OO, avril 2006, Kinshasa $)^{39}$.

À l'approche des élections et de l'instauration de la troisième République, l'attente d'un homme providentiel, dont la venue avait été annoncée par le chef spirituel Simon Kimbangu Kiangani, fut intensément rappelée. Le chef aurait parlé d'un « homme de l'ombre qui allait surgir ». Cette dernière expression, précisons-le, constitue un écho de la prophétie de Simon Kimbangu Kiangani selon laquelle « il y aura une surprise, un homme viendra ». Cet homme providentiel, de nombreux Kimbanguistes l'associent au ndayisua

37. U est un dignitaire administratif de l'Église, est âgé d'une soixantaine d'années, a fait des études universitaires et occupe un emploi dans l’Église.

38. <http ://www.kimbanguisme.net/bibliotheque/docs\&analyses/docs/art_20050627_bak.htm>

39. OO est un fidèle kimbanguiste, est âgé d'une quarantaine d'années, a fait des études secondaires supérieures et est sans emploi. 
( «le sommeil »), c'est-à-dire à ce qu'ils envisagent comme le moment de l' « enlèvement », $\mathrm{du}$ « grand sommeil de trois jours ». En somme à l'accomplissement eschatologique. L'homme providentiel s'identifie, de ce point de vue, à la figure eschatologique du nouveau « roi ». Contentons-nous ici de signaler que dans l'imaginaire kimbanguiste, le « roi » assumerait la totalité des « pouvoirs » conçus sous la forme de « trois pouvoirs » : le pouvoir spirituel, le pouvoir politique et le pouvoir scientifique ${ }^{40}$. Dans d'autres discours kimbanguistes, la complexité de cette image de l'homme providentiel s'articule, plus ou moins confusément, à celle de l'homme chargé de diriger la République démocratique du Congo. Les Kimbanguistes s'autorisent à cet égard d'une sentence laconique du chef spirituel, prophétisant que « celui qui dirigera ce pays sera un enfant de Dieu ». Un Kimbanguiste précise que cette prophétie signifie que « le pays sera dirigé par quelqu'un que Dieu choisira » (OO, avril 2006 Kinshasa $)^{41}$.

Davantage encore, la dimension théologico-politique semble culminer avec les croyances partagées aujourd'hui par la majorité des Kimbanguistes en l'incarnation de l'Esprit Saint et en une eschatologie réalisée. La croyance en l'identification de Kimbangu à l'Esprit Saint était présente à l'époque coloniale. Elle a persisté, clandestinement mais majoritairement, dans l'EJCSK jusqu'aux années 1990, en dépit du christocentrisme déclaré. Elle s'est ouvertement exprimée dès les années 1990, au lendemain du décès de Diangienda. À ce moment est aussi apparue la croyance en l'incarnation de la Trinité dans les trois fils de Kimbangu. Enfin, le nouveau chef spirituel depuis 2001, Simon Kimbangu Kiangani, l'aîné des petit-fils de Kimbangu, est officiellement ${ }^{42}$ désigné comme la réincarnation de son grand-père et, du même coup, comme l'incarnation de l'Esprit Saint. On est en présence de ce que Godelier appelait « un dieu vivant parmi les hommes » (Godelier 2007 : 207). Les Kimbanguistes partagent la conviction d'une eschatologie réalisée : la Jérusalem céleste serait déjà descendue dans leur ville sainte de Nkamba, mais elle resterait provisoirement invisible ${ }^{43}$. Kimbangu, ses trois fils et sa réincarnation, Simon Kimbangu Kiangani, y régneraient déjà.

La subtile dialectique de l'invisible et du visible qui fait l'efficace de l'imaginaire religieux du pentecôtisme (Corten et Mary 2000 : 17-21) vaut également, dans une large mesure, pour l'EJCSK. Mais ces convergences ne doivent pas occulter les points de

40. Cette croyance est intimement inséparable d'une prophétie que Kimbangu aurait faite, le 10 septembre 1921, à Mbanza-Nsanda, le jour où il aurait décidé de se livrer aux autorités coloniales belges.

41. OO est un fidèle kimbanguiste, est âgé d'une quarantaine d'années, a fait des études secondaires supérieures et est sans emploi.

42. Le slogan qui est dédié à l'actuel chef spirituel aujourd'hui est explicite : « Papa Simon Kimbangu Kiangani oyé (3 fois), Molimo Santu », ce qui signifie « Esprit Saint». Les fidèles ajoutent parfois à ce slogan : « Ebeto bien ebeto bien tozali na Nzambe na biso », ce qui signifie « Nous nous sentons bien car nous avons notre Dieu ».

43. L'invisible, à Nkamba, c'est Dieu, Kimbangu, les morts, la communauté des saints, ou encore les anges, en somme le royaume de Dieu. Mais il n'est pas négligeable de constater qu'il existe des degrés dans la perception de l'invisible. Certains fidèles kimbanguistes peuvent certes, occasionnellement, apercevoir une part de l'invisible. Mais le chef de l'Église kimbanguiste, est, lui, en prise permanente sur l'invisible. Entre lui et les fidèles, un seuil demeure, infranchissable. Autrement dit, la capacité exceptionnelle de perception de l'invisible trace une ligne de démarcation qui coïncide avec le privilège d'un pouvoir. 
divergence majeurs ${ }^{44}$. Ainsi la manière dont l'Esprit Saint se manifeste correspond de part et d'autre à « la topique de l'immanence » dont Corten constate la pertinence, au moins relative, pour les Églises pentecôtistes (Corten 2004). La convergence est loin d'être négligeable. Il reste cependant une différence considérable. C'est que cette immanence du religieux prend chez les Kimbanguistes une visibilité exceptionnelle. Le chef y est en effet identifié à l'Esprit Saint lui-même. Cette incarnation consonne avec d'autres concrétisations, en particulier la réalisation, au sens propre, de la Jérusalem céleste dans la ville sainte de Nkamba. L'EJCSK présente les traits d'une religion de l'immanence, mais sur un mode spécifique qui est celui de l'incarnation de l'Esprit Saint, et plus généralement, de l'invisible. Et cet invisible incarné est sans accointance avec l'invisible « deuxième monde », avec les « forces invisibles » comme « forces occultes » (Corten et Mary 2000 : 19), puisqu'il est une pleine positivité axiologique.

\section{Le statut politique de Nkamba : une concrétisation de l'imaginaire théologico-politique de l'EJCSK}

Depuis les années 1990, les Kimbanguistes nourrissent le projet que soit accordé à Nkamba, leur ville sainte située dans la Province du Kongo Central, un «statut international ». En 2006, ils ont introduit auprès du parlement congolais une demande de reconnaissance de souveraineté de Nkamba lui conférant un statut particulier. Le statut souhaité est celui d'un État autonome, forgé sur le modèle du Vatican. Un homme politique congolais de la mouvance présidentielle, qui était présent au Parlement quand la question y fut soulevée, explique son point de vue : prendre en compte une telle revendication équivaudrait à accepter « un démembrement du territoire national par renonciation [de l'État] à la souveraineté sur une partie de celui-ci »(HH, mai 2007, Kinshasa). Les parlementaires présents lors du débat auraient, dans leur ensemble, jugé qu'accorder à Nkamba quelque statut particulier que ce soit, risquait d'accentuer, dans la province du Kongo Central (ex-Bas-Congo), les revendications autonomistes ${ }^{45}$. La crainte que soit atteinte l'unité territoriale et politique du Congo atteste du caractère politico-religieux des revendications relatives à Nkamba.

La portée théologico-politique de Nkamba est d'ailleurs manifeste. Les Kimbanguistes considèrent cette ville comme ce qu'ils nomment souvent le centre ou le nombril du monde. Parler de nombril du monde, ce n'est pas seulement chercher à produire des effets métaphoriques. C'est viser à produire des « effets dans le réel » (Godelier 2007 ; Mélice 2007). C'est désigner le point vers lequel doivent téléologiquement converger toutes les nations. C'est aussi s'autoriser d'un projet hégémonique qui trouve son

44. Laissons ici en suspens la thèse de l'affaiblissement des Églises indépendantes depuis les années 1980 face aux nouvelles Églises charismatiques (Gifford 2004 : 170). Comme l'a avancé Gifford, le pentecôtisme africain des années 1990 n'est pas du même ordre que le pentecôtisme qui a pu inspirer les Églises Indépendantes antérieurement (Gifford $2001: 33$ ).

45. C'est plus particulièrement du Bundu dia Kongo (BDK) que le Parlement semble craindre une intensification de telles réactions. Aussi, alléguant le «manque de maturité politique des Congolais » (sic), le Parlement aurait décidé de ne pas signifier son refus à cette requête. La publicité de ce refus aurait entraîné le risque de réactions violentes, notamment du Bundu dia Kongo. Il n'est pas insignifiant que la Province du Kongo Central, lors des élections présidentielles, ait massivement plébiscité, comme tout le bloc Ouest, JeanPierre Bemba et qu'elle demeure, comme en témoignent notamment les actions (" province morte », etc.) du BDK, l'un des foyers d'opposition au nouveau gouvernement de Joseph Kabila. 
expression explicite dans une révélation attribuée à Kimbangu : «J'ai vaincu le monde et ses quatre coins m'ont été accordés par Dieu. À l'avenir, il n'y aura plus qu'une seule Église, une seule langue, un seul royaume et un seul roi, moi Simon Kimbangu ». La dimension théologico-politique de ces propos attribués à Kimbangu par l'historiographie kimbanguiste est patente. Théologique, puisqu'il s'agit de renverser le mouvement qui a mené à la dispersion consécutive à l'érection de Babel. Politique, puisqu'il s'agit dans le même temps de penser la relation du kimbanguisme à l'humanité comme une relation d'enveloppement.

\section{Conclusion}

Lorsque Bayart évoque le kimbanguisme, c'est pour donner l'exemple d'une Église indépendante tout autant que d'une Église accordée à la raison de l'État. Il n'est d'abord pas insignifiant qu'il parle à ce propos d'Églises dites indépendantes (Bayart 1989 : 117 et Bayart 1993 : 151). Cette précaution est loin d'être simplement rhétorique. Bayart se réclame en effet de T. Ranger pour stigmatiser l'erreur qui, exagérant l'africanité des unes et l'étrangeté des autres, incline à « croire que les Églises dites « indépendantes» sont plus « africaines » que l'Église catholique ou les Églises réformées intégrées au système protestant mondial » (Bayart 1993 : 132). C'est dire que le kimbanguisme n'échapperait pas au diagnostic que Bayart pose sur les Églises chrétiennes : elles auraient succombé à la force attractive de ce qu'il désigne comme la politique du ventre ${ }^{46}$. Davantage : non seulement le kimbanguisme offrirait, au plan religieux, l'exemple d'une adoption des « insignes symboliques de l'homo manducans » (Bayart 1993 : 151), mais il offrerait aussi, au plan politique, l'exemple d'une Église qui s'est accommodée du régime zaïrois. À cet égard, on pourrait être tenté de ranger le « kimbanguisme officiel » sous Mobutu parmi ces « théologies africaines qui déculpabilisent le pouvoir postcolonial ou exonèrent la délinquance d'État», et légitiment ainsi le «principe autoritaire » (Mbembe 1988 : 193). Nous avons cependant nuancé plus haut ce que ce type de diagnostic a de massif, de trop peu nuancé, voire de caricatural : c'est faire là peu de cas du principe kimbanguiste de respect des autorités, ainsi que de la contestation politique présente dans ce qu'Asch a appelé le "kimbanguisme des Kimbanguistes », c'est-à-dire des Kimbanguistes traditionalistes ou en total désaccord avec le mobutisme.

La critique acerbe de Bayart à l'encontre des christianismes africains donne d'autant plus de relief au lien que L'État en Afrique esquisse entre les sectes d'inspiration kimbanguiste contemporaines de Mobutu et les prophétismes de l'époque coloniale. Recourant à une problématique weberienne, il décrit leur détachement d'avec l'Église kimbanguiste « routinisée », alignée sur la raison d’État, aux marges de laquelle une fragmentation s'opère qui « alimente la dissidence sociale » (Bayart 1989 : 313). Il est intéressant de souligner que Bayart voit dans le retrait opéré par ces sectes, une volonté d'ignorer le pouvoir en place et non une véritable contestation de celui-ci. Nos entretiens attestent qu'au fond, cette conviction se retrouve au sein même de l'EJCSK officielle aujourd'hui : l'autorité théologico-politique reste incommensurable avec l'autorité politique effective. Et l'autorité politique effective, qu'on décide de la reconnaître ou de

46. Pour rappel : «Manger, c’est certes se nourrir, chose qui ne va pas de soi dans des économies de la rareté ou de la pénurie, en pleine phase d'un « ajustement structurel » des plus problématiques. C'est aussi accumuler, exploiter, vaincre, attaquer ou tuer en sorcellerie » (Bayart 1993 : 136). 
l'ignorer, apparaît indifférente à la législation divine. Bayart a évoqué " la création de véritables communautés théocratiques hors l'État [...] qui limitent et relativisent le champ étatique assurant ainsi une certaine revanche de la société sur celui-ci » (Bayart 1983 : 102-103). L'imaginaire kimbanguiste de l'EJCSK participe aujourd'hui partiellement de cette « revanche des sociétés africaines », de « leur sortie de l'État» (Mbembe 1988 : 22). Il resterait à comprendre comment cette forme de résistance de l'imaginaire kimbanguiste à l'État s'accommode de son obéissance à ce dernier et de son implication dans le processus électoral. Pour expliquer la relation paradoxale des Kimbanguistes au pouvoir politique effectif, on pourrait invoquer leur prudence, leur opportunisme peut-être. Mais, en plus profonde analyse, il s'agirait de rapporter leur position pratique à l'imaginaire kimbanguiste.

Ce paradoxe rencontre ce que, commentant Hannah Arendt, on a pu appeler « la condition paradoxale d'appartenance et de retrait par rapport au monde des phénomènes » (Taminiaux 2004, p.125-126). Dans le cas du kimbanguisme actuel, il trouve sa résolution dans l'historicisme millénariste. L'excès incommensurable du projet théologico-politique sur la réalité politique présente, c'est à l'histoire qu'il appartiendrait de le combler. Téléologique, l'historicité que supporte l'imaginaire kimbanguiste opère une résolution immanente du paradoxe. Dans cette perspective millénariste, les prophéties constituent autant de signes annonciateurs du dénouement à venir. Et il n'est pas négligeable que certains Kimbanguistes reconnaissent dans la prophétie kimbanguiste de l'homme providentiel la figure de Joseph Kabila et voient en lui une attestation du sens théologicopolitique de l'Histoire. Rapportés à ce dernier, les engagements politiques effectifs restent marqués du sceau du provisoire et de l'attente eschatologique.

\section{Références citées}

AkIEle, Basile. « Papa Diangienda Kuntima, l'éducateur multidimensionnel », <http ://www.kimbanguismeejcsk.org>

Амм,

$1^{\text {er }}$ août 2005. « S.E. Simon Kimbangu Kiangani très rassurant : 'L'Église est comme une mer qu'on ne peut vider d'eau' », Le Kimbanguisme Info, 66, p. 3.

26 septembre 2005. «Le couple S.E. Simon Kimbangu Kiangani s'est enrôlé le dimanche 14 août à Nkamba », Le Kimbanguisme Info, 68, p. 2.

Asch, Susan, 1983. L'Église du prophète Kimbangu. De ses origines à son rôle actuel au Zaïre. Paris : Karthala.

Balandier, Georges, 1982 [1955]. Sociologie actuelle de l'Afrique noire. Dynamique sociale en Afrique centrale. Paris : Quadrige/PUF.

Banda-Mwaka, Justin, mars-juin 1971. « Le kimbanguisme en tant que mouvement prépolitique chez les Kongo », Problèmes sociaux congolais, 92-93, p. 3-54.

Bandzouzi, Alphonse, 2002. Le kimbanguisme. Paris : Jouve.

BAYART, Jean-François,

1983. « La revanche des sociétés africaines », Politique africaine, 11, p. 95-128.

1989. L'État en Afrique. La politique du ventre. Paris : Fayard.

1993. Religion et modernité politique en Afrique noire. Dieu pour tous et chacun pour soi. Paris : Karthala.

1996. L'illusion identitaire. Paris : Fayard. 
Bayart, Jean-François, Achille Mbembe, Comi Toulabor, 2008 [1992]. Le politique par le bas en Afrique noire. Paris : Karthala.

Bernhard, Wendy Lee, 1999. Bundu Dia Kongo : A New Non-Christian Religious Movement in Zaïre. Michigan : UMI Dissertation Services.

Corten, André et André MARY, (éds), 2000. Imaginaires politiques et pentecôtismes. Afrique/Amérique latine. Paris : Karthala.

CORTEN, André, 2004. « Le nouveau religieux pentecôtiste. Éléments d'explication politico-théologique », PentecoStudies, vol. 3, 2, 17 p. <www.glopent.net/pentecostudies/2004/corten 2004.pdf>

CRISP, 1960. Le Kimbanguisme, Courrier hebdomadaire du 8 janvier 1960.

De Boeck, Filip et Marie-Françoise Plissart, 2005. Kinshasa. Récits de la ville invisible. Bruxelles : La Renaissance du Livre.

De Villers, Gauthier,

1995. De Mobutu à Mobutu. Trente ans de relations Belgique-Zaïre. Bruxelles : De Boeck.

1998. « Identifications et mobilisations politiques au Congo-Kinshasa », Politique africaine, 72, p.81-97.

Devisch, René,

1996. " "Le pillage de Jésus" : les églises de guérison et la villagisation de Kinshasa », in Gauthier de Villers (sous dir.), Phénomènes informels et dynamiques culturelles en Afrique, p. 91-138. Cahiers africains, 19-20.

1998. «La parodie dans les Églises de guérison à Kinshasa », Itinéraires et contacts de culture, 25, p. 115-174.

2000. «Les Églises de guérison à Kinshasa. Leur domestication de la crise des institutions », in André Corten et André Mary (éds), Imaginaires politiques et pentecôtismes, p. 119-141. Paris : Karthala.

Diangienda Kuntima, Joseph, 1984. L'histoire du kimbanguisme. Kinshasa : Éditions kimbanguistes.

Droogers, André, 1980. « Kimbanguism at the Grass Roots. Beliefs in a Local Kimbanguist Church », Journal of Religion in Africa, vol. XI, fasc. 3, p. 188-211.

EJCSK, 2007. Actes de la Conférence internationale sur Simon Kimbangu, l’Envoyé spécial de notre Seigneur Jésus-Christ. France : Éditions kimbanguistes-EKI.

GIFFORD, Paul,

2001. African Christianity. Its Public Role. London : Hurst \& Company.

2004. « Persistence and Change in Contemporary African Religion », Social Compass, 51 (2), p. 169176.

Godelier, Maurice, 2007. Au fondement des sociétés humaines. Paris : Albin Michel.

Jourdan, François, 2006. « Le Jeune sage », Jeune Afrique du 26 février 2006, <http ://www.jeuneafrique.com>.

LKI, 26 décembre 2005. " Référendum constitutionnel : le Chef Spirituel remplit son devoir civique ", Le Kimbanguisme Info, 72, p. 3.

Luntadila Ndala Za Fwa, Lucien, 1990. Qui est Papa Diangienda?. Kinshasa : Édition Lula.

MaC Gaffey, Wyatt, 1992. « Kimbanguism in the Independence Process », Recueil d'études " Congo 19551960». Bruxelles : Académie Royale des Sciences d'Outre-Mer, p. 329-342.

Malenge, K.M., 1992. Prêtre dans la rue. Kinshasa : Éditions Baobab.

Mambu-Ma-Mwana, $1^{\text {er }}$ août 2005. « Le mariage est scellé entre l'Église kimbanguiste et la Commission électorale Indépendante (CEI). L’Abbé-Président Malu-Malu rassuré du soutien des Kimbanguistes pour l'aboutissement heureux du processus électoral », Le Kimbanguisme Info, 66, p. 5.

Manny, K.Y., 16-23 juillet 1992. « Diangienda, le message était destiné à Mobutu », La Gazette, 6, p.3. 
Martin, Marie-Louise, 1981. Kimbangu. Un prophète et son Église. Lausanne : Éditions du Soc.

Mвеmbe, Achille, 1988. Afriques indociles. Christianisme, pouvoir et État en société postcoloniale. Paris : Karthala.

MÉLICE, Anne,

2001. "Le kimbanguisme : un millénarisme dynamique de la terre aux Cieux », in Jan-Lodewijk Grootaers (ed.), Millenarian Movements in Africa and the Diaspora/Mouvements millénaristes en Afrique et dans la diaspora, p. 35-54. Bulletin des Séances, Académie Royale des Sciences d'Outre-Mer, 47 (suppl.).

2006. «Un terrain fragmenté : le kimbanguisme et ses ramifications », Civilisations, LIV (1-2), p. 67-76.

2007. " Le primat de l'imaginaire sur le symbolique dans l'anthropologie de Maurice Godelier », in Joseph Denooz, Véronique Dortu et Rudy Steinmetz, Mosä̈ques. Hommages à Pierre Somville, p. 173-180. Liège : CIPL.

À paraître. " La désobéissance civile des Kimbanguistes face à la violence coloniale (1921-1959) ", communication inédite à la conférence internationale Colonial Violence in Congo / La Violence coloniale au Congo, organisée par l'Association belge des Africanistes et par le Musée Royal de l'Afrique centrale de Tervuren, Tervuren, 12 et 13 mai 2005.

NDAYwel È NZIEM, Isidore,

1993. « La société zaïroise dans le miroir de son discours religieux (1990-1993) », Cahiers africains, 6, $102 \mathrm{p}$.

1998. Histoire générale du Congo. Bruxelles : De Boeck.

Ne Muanda Nsemi,

1991. Allez à Mama Yemo. Kinshasa : Éditions Mpolo Ngimbi.

1993. Non, Mr le Professeur. Kinshasa : Éditions Mpolo Ngimbi.

1995. Bukongo 1. Kinshasa : Éditions Mpolo Ngimbi.

1998. Vérité et réconciliation. Kinshasa : Éditions Mpolo Ngimbi.

NZAZI MabidI, Marcel, 2006. « Le destin exceptionnel de Joseph Kabila », <http ://www.presidentrdc.cd/ edito0106.html>

Passy Mriosso, S., 1998. « La prophétie du prophète Simon Kimbangu sur l'avenir politique de la République démocratique du Congo faite le 17 juin 1944 dans la prison d'Élisabethville », La Renaissance, 645, 29 sept.-15 oct. 1998), <http :/www.ejcsk-france.com/ELISABETHVILLE17juin_1944.html> et $<$ http ://www.radio-simonkimbangu.com $>$.

Safou Sindani, Ch., 11-12-13 juillet 1992. «Les Kimbanguistes et le processus démocratique », Elima, 96, p. 3 et 8 .

Taminiaux, Jacques, 2004. « Le paradoxe de l'appartenance et du retrait », in Colloque Hannah Arendt, Politique et pensée, p. 121-143. Paris : Petite Bibliothèque Payot.

Tosel, André, 1995. " La loi et ses législateurs ou les avatars du théologico-politique », in Démocratie et libéralismes, p. 177-195. Paris : Kimé.

Ustorf, Werner, 1975. Africanische Initiative. Das Aktive Leiden des Propheten Simon Kimbangu. Frankfurt : Herbert Lang.

VAn Lierde, Jean, 1986. «Jean-Lasserre et la non-violence kimbanguiste », Cahiers de la Réconciliation, 3, p. 19-31.

Young, Crawford, 1965. Politics in the Congo. Princeton-New Jersey : Princeton University Press. 